Ophthalmologe 2012 $\cdot 109: 529-530$

DOI 10.1007/s00347-011-2505-8

C) Springer-Verlag 2012

\section{N. Stübiger}

Universitäts-Augenklinik,Uveitis Zentrum, Campus Virchow Klinikum und

Campus Benjamin Franklin, Universitätsmedizin Berlin, Charité, Berlin

\title{
Morbus Behçet
}

\section{Epidemiologie - klinische Manifestationen - Immunologie und Therapie}

Beim Morbus Behçet (MB) handelt es sich um eine komplexe, rezidivierende systemische Vaskulitis, deren Ätiologie bis heute nicht geklärt ist. Der Verlauf der Erkrankung ist sehr variabel, in vielen Fällen jedoch chronisch progredient. Nahezu immer sind verschiedene Organsysteme beteiligt, insbesondere die Haut, die Schleimhäute und die Augen.

Zwar handelt es sich beim MB um eine Erkrankung, die schon lange bekannt ist, jedoch hat sie bis heute noch viele Fragen offen gelassen. Bereits Hippokrates (460370 v. Chr.) erwähnte das Krankheitsbild in der Antike in seinem 3. EpidemionBuch, und bis zum ausgehenden 19. Jahrhundert wurde der MB häufig als Tuberkulose oder als Syphilis verkannt.

Die ersten (treffenden) Beschreibungen gehen auf Benediktos Adamantiades und Hulusi Behçet zurück. Der griechische Ophthalmologe Benediktos Adamantiades (1875-1962) beschrieb 1930 auf einem Kongress der medizinischen Gesellschaft einen Patienten mit einer Hypopyoniritis und oralen sowie genitalen Aphthen und einer Arthritis. Dies berichtete er 1931 in den Annales d'Oculistique [1]. Der türkische Dermatologe $\mathrm{Hu}$ lusi Behçet (1889-1948) publizierte 1937 in der Zeitschrift Dermatologische Wochenschrift einen Artikel „Über rezidivierende aphthoese, durch einen Virus verursachte Geschwüre an Mund, am Auge und an den Genitalien“" [2]. Obwohl Adamantiades wenige Jahre vor Behçet seine Beobachtungen mitgeteilt hatte, wurde die Krankheit 1947 während eines internationalen medizinischen Kongresses in Genf als „Morbus Behçet“ benannt. Even- tuell lag es daran, dass Adamantiades' $\mathrm{Pu}$ blikation in Französisch erschienen war, sodass sich zunächst in der amerikanischen Literatur der Begriff „Behcet’s syndrome" durchsetzte. Heute wird der Terminus Morbus Behçet oder aber Morbus Adamantiades-Behçet gleichermaßen verwendet, um den wissenschaftlichen Leistungen sowohl von Adamantiades als auch von Behçet gerecht zu werden.

\section{》) Beim Morbus Behçet handelt es sich um eine komplexe, rezidivierende systemische Vaskulitis}

Damit Willkommen zum Leitthemenheft "Morbus Behçet“ in der aktuellen Ausgabe der Zeitschrift Der Ophthalmologe. Wir haben einige aktuelle Aspekte herausgegriffen und möchten den Leser hiermit auf dieses wichtige Krankheitsbild aufmerksam machen.

Hinweise auf die Epidemiologie und Verteilung des MB geben Altenburg et al. (Klinik für Dermatologie, Venerologie und Allergologie, Immunologisches Zentrum, Städtisches Klinikum Dessau) wie auch Kneifel et al. (Klinik für Augenheilkunde, Städtisches Klinikum Dessau). Sie heben insbesondere die ophthalmologischen und die sonstigen klinischen Unterschiede zwischen in Deutschland lebenden Türken und an MB erkrankten Deutschen hervor. Interessanterweise fanden sie eine Prävalenz des MB von 0,4:100.000 unter den Einwohnern Berlins deutscher Herkunft - dies ist auf einem vergleichbar niedrigen Niveau wie in Großbritan- nien oder in den USA - während die Autoren für die türkische Bevölkerung Berlins eine Prävalenz von 77,4:100.000 finden konnten, die vergleichbar ist mit dem europäischen Teil der Türkei. Im Gegensatz zu früheren Studien konnten sie also zeigen, dass die Erkrankungshäufigkeit in Berlin identisch ist mit den Zahlen aus der Türkei. Damit tritt der früher als ursächlich für den MB vermutete Umweltfaktor eher in den Hintergrund, und die genetische Komponente (HLA-B 51) wird deutlich in den Vordergrund gerückt.

Die Problematik der Diagnosestellung wird durch die Arbeitsgruppe von Kötter et al. aus der Medizinischen und Neurologischen Klinik der Universität Tübingen beleuchtet. Durch die sehr variable Ausprägung der klinischen Symptome wird der MB häufig nicht nur äußerst spät, sondern oft auch fehldiagnostiziert. In diesem Beitrag werden wertvolle Hinweise und Beschreibungen mit einem Schwerpunkt auf den rheumatologisch/internistischen und neurologischen Manifestationen sowie auf die Auswahl der entsprechenden Diagnosekriterien gegeben.

Oft steht die ophthalmologische Beteiligung im Vordergrund. Zum Teil gibt es für den MB sehr typische okuläre Veränderungen, die im „diagnostischen Dschungel“ dieser systemischen Vaskulitis durchaus richtungsweisend sein und damit zu einer schnelleren Diagnosestellung führen können. Einen Überblick über typische - aber auch über eher untypische - ophthalmologische Veränderungen gibt der Beitrag von Stübiger et al. (Uveitis Zentrum der Augenklinik der Charite, Universitätsmedizin Berlin) 
Die Ätiologie des MB gibt bis zum heutigen Tage Rätsel auf und ist nach wie vor ungeklärt. Jedoch ist unser Verständnis v. a. zu den immunologischen Veränderungen dieses Erkrankungsbildes in den letzten Jahren deutlich gewachsen. Pleyer et al. (Uveitis Zentrum der Augenklinik der Charite, Universitätsmedizin Berlin) stellen dies ausführlich dar und zeigen, dass aus diesen Erkenntnissen heraus bereits neue Therapiemöglichkeiten entstanden sind.

Die bedeutend höhere Effektivität dieser neuen Therapieansätze, insbesondere die der Biologika, wird durch den Beitrag von Deuter et al. (Augenklinik der Universität Tübingen) anschaulich vermittelt. Vor allem die Therapie des okulären MB stellte bis vor Kurzem ein große Herausforderung dar, da noch bis zum Ende des letzten Jahrhunderts trotz des therapeutischen Einsatzes konventioneller immunsuppressiver Substanzen eine Erblindung in bis zu 70\% der an MB Erkrankten drohte. Diese äußerst schlechte Visusprognose konnte jedoch durch den Einsatz der neuen, in diesem Beitrag vorgestellten Substanzen grundlegend verbessert werden.

Wir möchten uns bei den Autoren bedanken und hoffen, mit der Auswahl der Beiträge den Lesern einen aktuellen Überblick sowie einen anschaulichen Einblick in ein spannendes Feld der Augenheilkunde bieten zu können.

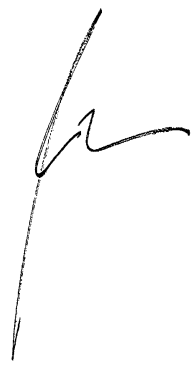

N. Stübiger

\section{Korrespondenzadresse}

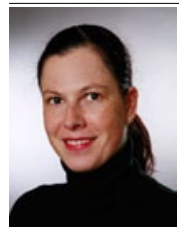

Prof. Dr. N. Stübiger

Universitäts-Augenklinik,Uveitis Zentrum, Campus Virchow Klinikum und Campus Benjamin Franklin, Universitätsmedizin Berlin, Charité Hindenburgdamm 30, 12200 Berlin nicole.stuebiger@charite.de

\section{Literatur}

1. Adamantiades B (1931) Sur un cas d'iritis hypopyon recidivant. Ann Oculist 168:271-278

2. Behçet $H$ (1937) Über rezidivierende, aphtoese, durch einen Virus verursachte Geschwüre im Mund, an den Augen und an den Genitalien. Dermatol Wochenschr 105:1152-1157

\section{Behandlung von Infektionen kann Augen schädigen}

Medikamente gegen Infektionen können Sehstörungen auslösen, warnt die Deutsche Gesellschaft für Ophthalmologie (DOG). Um dauerhafte Schäden an den Augen zu vermeiden, sollten Patienten Sehstörungen beachten und sich rechtzeitig vom Augenarzt untersuchen lassen. Medikamente gegen Infektionen erreichen über den Kreislauf auch die Augen. Funktionsstörungen können dann durch kleinste Veränderungen auftreten. Nebenwirkungen von Arzneimitteln am Auge entwickeln sich jedoch oft langsam. Die Patienten sprechen deshalb den Arzt erst spät darauf an. Um Schäden zu vermeiden, müsste das medizinische Personal auf Sehstörungen achten und gezielt danach fragen. Bei einigen Medikamenten sollten routinemäßig augenärztliche Untersuchungen erfolgen.

Sehr häufig treten Sehstörungen bei der Behandlung von Pilzinfektionen mit Voriconazol auf. Diese können verschwommenes Sehen, Störungen des Farbensehens, oder Scheue vor Licht umfassen. Die Störungen gehen meist innerhalb von einer Stunde zurück.

Das Antibiotikum Telithromycin, welches zur Behandlung von bakteriellen Infektionen und auch bei Lungenentzündungen eingesetzt wird, kann die Motorik der Augen stören. Dies verzögert mitunter den Wechsel von Fern- auf Nahsicht oder erzeugt Doppelbilder.

Das Antibiotikum Linezolid kann dauerhafte Schäden des Sehnervs verursachen - bis hin zum Verlust des Sehvermögens. Dieses Antibiotikum kommt bei Infektionen durch den Problemkeim MRSA (Methicillin-resistenter Staphylococcus aureus) zum Einsatz. Vor allem bei längerer Therapiedauer könne es zu dieser Neuropathie kommen. Da bestimmte Medikamente gegen Infektionen zu Sehstörungen führen, seien Nebenwirkungen am Auge frühzeitig in die Therapieplanung einzubeziehen. Augenärztliche Kontrollen seien möglichst vorab zu planen.

Literatur:

Huber M, Stahlmann R (2012) Arzneimittelnebenwirkungen am Auge bei systemischer Therapie mit Antiinfektiva. Deutsche Medizinische Wochenschrift 137:85-89

\section{Quelle:} Deutsche Ophthalmologische Gesellschaft (DOG), www.dog.org 